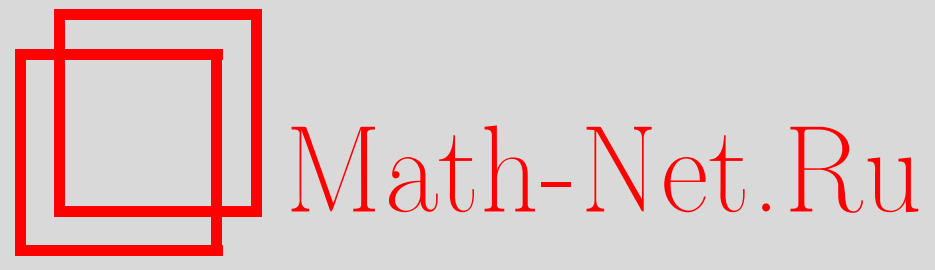

А. А. Грушо, Е. Л. Шумицкая, Модель невлияния и скрытые каналы, Дискрет. матем., 2002, том 14, выпуск 1, 1116

DOI: https://doi.org/10.4213/dm227

Использование Общероссийского математического портала Math-Net.Ru подразумевает, что вы прочитали и согласны с пользовательским соглашением http://www.mathnet.ru/rus/agreement

Параметры загрузки:

IP : 3.91 .87 .62

26 апреля 2023 г., 17:00:10

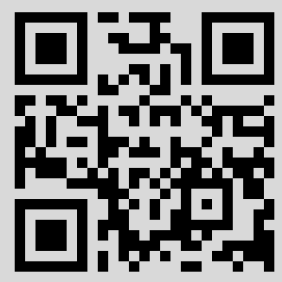




\title{
Модель невлияния и скрытые каналы
}

\author{
(C) 2002 г. А. А. Грушо, Е. Л. Шумицкая
}

В статье рассматриваются математические модели безопасных систем, а именно, традиционная модель невлияния и модель невлияния по вероятности. Доказано, что система может удовлетворять условиям традиционной модели невлияния, но при этом существует скрытый канал с верхнего уровня на нижний. Найдено достаточное условие отсутствия в автоматной модели системы скрытых каналов.

Работа выполнена при поддержке Российского фонда фундаментальных исследований, проект 01-01-00895.

Будем считать, что теоретико-автоматные модели достаточно хорошо отражают исследуемые свойства в реальных системах. Пусть компьютерная система моделируется абстрактным автоматом $A=(X, S, Y, \delta, \lambda)$, где $X, S, Y$ - конечные непустые множества, соответственно, входной алфавит, множество состояний и выходной алфавит, $\delta: X \times A \rightarrow S-$ функция переходов, $\lambda: X \times A \rightarrow Y-$ функция выходов.

Будем рассматривать следующую автоматную модель двухуровневой компьютерной системы.

Пусть $X=X_{H} \times X_{L}$, где $X_{H}$ и $X_{L}$ - множества входов для пользователей уровней $H$ (High) и $L$ (Low), соответственно. Далее везде будем предполагать, что имеется только один пользователь каждого уровня, и называть их $H$ и $L$, соответственно. Аналогично, пусть $S=S_{H} \times S_{L}, Y=Y_{H} \times Y_{L}$. Будем считать, что множества $X_{H}, X_{L}, Y_{H}, Y_{L}$ могут содержать среди своих элементов пустое слово, которое мы будем обозначать $e$.

Определение 1. Пусть $s^{1}, s^{2} \in S$. Определим отношение эквивалентность $\sim$, полагая $s^{1} \sim s^{2}$, если $s_{L}^{1}=s_{L}^{2}$. Обозначим $S / \sim$ фактор-множество относительно отношения $\sim$, класс эквивалентности, содержащий $s$, будем обозначать $[s], s \in S$.

Аналогично определим отношение эквивалентности на множестве выходов $Y$.

Определение 2. Пусть $y^{1}, y^{2} \in Y$. Определим отношение эквивалентности $\sim$, полагая $y^{1} \sim y^{2}$, если $y_{L}^{1}=y_{L}^{2}$. Элементы фактор-множества $Y / \sim$ будем обозначать $[y]$.

В силу различия контекстов путаницы с одинаковым обозначением отношения эквивалентности не возникнет.

Рассмотрим простейшую теоретико-вероятностную модель невлияния. Заметим, что некоторые наши определения несколько отличаются от традиционных [1], мы будем говорить о невидимости верхнего уровня с нижнего уровня. Однако по сути эта модель близка к модели невлияния [1]. 
Определение 3. Будем говорить, что $L$ не видит $H$, если функция

$$
\tilde{\delta}: X_{L} \times S / \sim \rightarrow S / \sim
$$

определяемая формулой

$$
\tilde{\delta}\left(x_{L},[s]\right)=\left[\delta\left(\left(x_{H}, x_{L}\right), s^{\prime}\right)\right], \quad x_{H} \in X_{H}, \quad s^{\prime} \in[s],
$$

и функция $\tilde{\lambda}: X_{L} \times S / \sim \rightarrow Y / \sim$, определяемая формулой

$$
\tilde{\lambda}\left(x_{L},[s]\right)=\left[\lambda\left(\left(x_{H}, x_{L}\right), s^{\prime}\right)\right], \quad x_{H} \in X_{H}, \quad s^{\prime} \in[s]
$$

корректно определены.

Система, удовлетворяющая определению 3 , традиционно считается безопасной.

Лемма 1. Пусть $L$ не видит $H$ и фихсировань $y_{L}^{0} \in Y_{L}, u s_{L}^{0} \in S_{L}$. Тогда для всех $x_{H}^{\prime}, x_{H}^{\prime \prime} \in X_{H} u$ всех $s_{H}^{\prime}, s_{H}^{\prime \prime} \in S_{H}$

$$
\begin{aligned}
\left\{x_{L} \in X_{L}: \lambda\left(\left(x_{H}^{\prime}, x_{L}\right)\right.\right. & \left.\left.,\left(s_{H}^{\prime}, s_{L}^{0}\right)\right)=\left(y_{H}, y_{L}^{0}\right), y_{H} \in Y_{H}\right\} \\
& =\left\{x_{L} \in X_{L}: \lambda\left(\left(x_{H}^{\prime \prime}, x_{L}\right),\left(s_{H}^{\prime \prime}, s_{L}^{0}\right)\right)=\left(y_{H}, y_{L}^{0}\right), y_{H} \in Y_{H}\right\}
\end{aligned}
$$

Доказательство. Так как $L$ не видит $H$, функция $\tilde{\lambda}$ корректно определена. Это значит, что если для некоторого $x_{L}^{\prime} \in X_{L}$

$$
\lambda\left(\left(x_{H}, x_{L}^{\prime}\right),\left(s_{H}, s_{L}^{0}\right)\right)=\left(y_{H}, y_{L}^{0}\right),
$$

для некоторых $x_{H} \in X_{H}, s_{H} \in S_{H}, y_{H} \in Y_{H}$, то для всех $x_{H}^{\prime} \in X_{H}$ и $s_{H}^{\prime} \in S_{H}$ существует $y_{H} \in Y_{H}$ такое, что

$$
\lambda\left(\left(x_{H}^{\prime}, x_{L}^{\prime}\right),\left(s_{H}^{\prime}, s_{L}^{0}\right)\right)=\left(y_{H}, y_{L}^{0}\right)
$$

В рассматриваемых множествах $y_{H}$ пробегает все свои значения, поэтому лемма доказана.

Следствие 1. Пусть $L$ не видит $H$. Тогда для любых фиксированнъх $y_{L}^{0} \in Y_{L}$, $s_{L}^{0} \in S_{L}$ имеет место равенство

$$
\begin{aligned}
& \left\{\left(x_{H}, x_{L}\right) \in X: \lambda\left(\left(x_{H}, x_{L}\right),\left(s_{H}^{\prime}, s_{L}^{0}\right)\right)=\left(y_{H}, y_{L}^{0}\right), y \in Y_{H}\right\} \\
& \quad=\left\{\left(x_{H}, x_{L}\right) \in X: \lambda\left(\left(x_{H}, x_{L}\right),\left(s_{H}^{\prime \prime}, s_{L}^{0}\right)\right)=\left(y_{H}, y_{L}^{0}\right), y_{H} \in Y_{H}\right\}
\end{aligned}
$$

$\partial \Omega я$ всех $s_{H}^{\prime}, s_{H}^{\prime \prime} \in S_{H}$.

Утверждение непосредственно следует из леммы.

Хотя пользователю $L$ неизвестно сообщение $x_{H}$, ему может быть известна статистическая связь между входными сообщениями $x_{L}$ и $x_{H}$ или известно априорное совместное распределение векторов $\left(x_{L}, x_{H}\right)$. В этих случаях возможен скрытый канал с верхнего уровня на нижний. Чтобы доказать существование скрытого канала, определим невидимость в статистическом смысле.

Пусть на множестве $X=X_{H} \times X_{L}$ задано вероятностное распределение Р. Для простоты будем считать, что в $X$ нет таких элементов, вероятность которых равна 
нулю. Распределение $\mathrm{P}$ индуцирует на множествах $X_{H}$ и $X_{L}$ распределения вероятностей $\mathbf{P}_{H}$ и $\mathbf{P}_{L}$ :

$$
\begin{aligned}
& \mathbf{P}_{H}\left\{x_{H}\right\}=\mathbf{P}\left(\left\{\left(x_{H}, x_{L}\right): x_{L} \in X_{L}\right\}\right)=\sum_{x_{L} \in X_{L}} \mathbf{P}\left(x_{H}, x_{L}\right), \\
& \mathbf{P}_{L}\left\{x_{L}\right\}=\mathbf{P}\left(\left\{\left(x_{H}, x_{L}\right): x_{H} \in X_{H}\right\}\right)=\sum_{x_{H} \in X_{H}} \mathbf{P}\left(x_{H}, x_{L}\right) .
\end{aligned}
$$

С помощью функции $\lambda$ при каждом фиксированном состоянии $s$ распределение $\mathbf{P}$ индуцирует на $Y$ распределение вероятностей $\mathbf{Q}^{s}$ такое, что

$$
\mathbf{Q}^{s}(y)=\mathbf{P}\{x \in X: \lambda(x, s)=y\} .
$$

Аналогично формуле (4) получаем, что

$$
\begin{aligned}
\mathbf{Q}_{L}^{s}\left\{y_{L}\right\} & =\mathbf{Q}^{s}\left(\left\{\left(y_{H}, y_{L}\right): y_{H} \in Y_{H}\right\}\right) \\
& =\sum_{y_{H} \in Y_{H}} \mathbf{Q}^{s}\left(y_{H}, y_{L}\right)=\sum_{y_{H} \in Y_{H}} \mathbf{P}\left\{x \in X: \lambda(x, s)=\left(y_{H}, y_{L}\right)\right\} \\
& =\mathbf{P}\left\{x \in X: \lambda(x, s)=\left(y_{H}, y_{L}\right), y_{H} \in Y_{H}\right\}
\end{aligned}
$$

Лемма 2. Пусть $L$ не видит $H$ в смысле определения 3. Тогда для любых двух эквивалентных состояний распределения вероятностей на $Y_{L}$ совпадаю, то есть для всех $y_{L} \in Y_{L} u s^{1} \sim s^{2}$

$$
\mathbf{Q}_{L}^{s^{1}}\left\{y_{L}\right\}=\mathbf{Q}_{L}^{s^{2}}\left\{y_{L}\right\}
$$

Доказательство. Так как $s^{1} \sim s^{2}$, их $L$-компоненты совпадают. По формуле (5)

$$
\begin{aligned}
& \mathbf{Q}_{L}^{s^{1}}\left\{y_{L}\right\}=\mathbf{P}\left\{\left(x_{H}, x_{L}\right) \in X: \lambda\left(\left(x_{H}, x_{L}\right),\left(s_{H}^{1}, s_{L}\right)\right)=\left(y_{H}, y_{L}\right), y_{H} \in Y_{H}\right\} \\
& \mathbf{Q}_{L}^{s^{2}}\left\{y_{L}\right\}=\mathbf{P}\left\{\left(x_{H}, x_{L}\right) \in X: \lambda\left(\left(\left(x_{H}, x_{L}\right),\left(s_{H}^{2}, s_{L}\right)\right)=\left(y_{H}, y_{L}\right), y_{H} \in Y_{H}\right\}\right.
\end{aligned}
$$

А так как $L$ не видит $H$, по следствию 1

$$
\begin{aligned}
\left.\left\{x_{H}, x_{L}\right\} \in X: \lambda\left(\left(x_{H}, x_{L}\right),\left(s_{H}^{1}, s_{L}\right)\right)=\left(y_{H}, y_{L}\right), y_{H} \in Y_{H}\right\} & \\
= & \left\{\left(x_{H}, x_{L}\right) \in X: \lambda\left(\left(x_{H}, x_{L}\right),\left(s_{H}^{2}, s_{L}\right)\right)=\left(y_{H}, y_{L}\right), y_{H} \in Y_{H}\right\}
\end{aligned}
$$

Таким образом, $\mathbf{Q}_{L}^{s^{1}}\left\{y_{L}\right\}=\mathbf{Q}_{L}^{s^{2}}\left\{y_{L}\right\}$. Лемма доказана.

Далее определим совместную вероятность на $X_{H} \times Y_{L}$ при каждом состоянии $s \in S$, по формуле

$$
\mathbf{P}^{s}\left(x_{H}^{0}, y_{L}^{0}\right)=\mathbf{P}\left\{\left(x_{H}^{0}, x_{L}\right): x_{L} \in X_{L}, \lambda\left(\left(x_{H}^{0}, x_{L}\right), s\right)=\left(y_{H}, y_{L}\right), y_{H} \in Y_{H}\right\}
$$

Пользуясь этой совместной вероятностью, условную вероятность можно записать в виде

$$
\mathbf{P}^{s}\left(y_{L} \mid x_{H}\right)=\frac{\mathbf{P}^{s}\left(x_{H}, y_{L}\right)}{\mathbf{P}_{H}\left(x_{H}\right)}
$$


Лемма 3. Пусть $L$ не видит $H$. Тогда для любых двух эквивалентных состояний условные распределения, определяемъе формулой (7), совпадают, то есть

$$
\mathbf{P}^{s^{1}}\left(y_{L} / x_{H}\right)=\mathbf{P}^{s^{2}}\left(y_{L} / x_{H}\right), \quad y_{L} \in Y_{L}, \quad x_{H} \in X_{H}, \quad s^{1} \sim s^{2} .
$$

Доказательство. Так как $s^{1} \sim s^{2}$, их $L$-компоненты совпадают. По формуле (6)

$$
\begin{aligned}
& \left.\mathbf{P}^{s^{1}}\left(x_{H}, y_{L}\right)=\mathbf{P}\left\{\left(x_{H}, x_{L}\right): x_{L} \in X_{L}, \lambda\left(\left(x_{H}, x_{L}\right),\left(s_{H}^{1}, s_{L}\right)\right)=\left(y_{H}, y_{L}\right), y_{H} \in Y_{H}\right\}\right), \\
& \mathbf{P}^{s^{2}}\left(x_{H}, y_{L}\right)=\mathbf{P}\left(\left\{\left(x_{H}, x_{L}\right): x_{L} \in X_{L}, \lambda\left(\left(x_{H}, x_{L}\right),\left(s_{H}^{2}, s_{L}\right)\right)=\left(y_{H}, y_{L}\right), y_{H} \in Y_{H}\right\}\right) .
\end{aligned}
$$

А так как $L$ не видит $H$, по лемме 1 множества под знаком вероятности совпадают, то есть

$$
\mathbf{P}^{s^{1}}\left(x_{H}, y_{L}\right)=\mathbf{P}^{s^{2}}\left(x_{H}, y_{L}\right)
$$

Теперь, применяя формулу (7), получаем требуемое равенство. Лемма доказана.

Определение 4. Пусть $L$ не видит $H$. Будем говорить, что $L$ не видит $H$ по вероятности, если для всех $x_{H} \in X_{H}, y_{L} \in Y_{L},[s] \in S / \sim$

$$
\mathbf{P}^{s^{\prime}}\left(y_{L} / x_{H}\right)=\mathbf{Q}^{s^{\prime \prime}}\left(y_{L}\right), \quad s^{\prime}, s^{\prime \prime} \in[s]
$$

Корректность этого определения следует из лемм 2 и 3.

Теперь на простом примере покажем, что невидимости в смысле определения 3 недостаточно для невидимости по вероятности.

Пример 1. Пусть $X_{H}=X_{L}=\{0,1, e\}$, где $e-$ пустое слово, $S_{H}=\left\{s_{H}^{1}, s_{H}^{2}\right\}$, $S_{L}=\left\{s_{L}^{1}, s_{L}^{2}\right\}, Y_{H}=Y_{L}=\{0,1, e\}$. Распределение вероятностей зададим следующим образом:

\begin{tabular}{c|ccccccccc}
$\left\{x_{H}, x_{L}\right\}$ & $(0,0)$ & $(0,1)$ & $(1,0)$ & $(1,1)$ & $(0, e)$ & $(e, 0)$ & $(1, e)$ & $(1, e)$ & $(e, e)$ \\
\hline $\mathbf{P}\left(x_{H}, x_{L}\right)$ & $1 / 9$ & $1 / 9$ & $1 / 18$ & $1 / 6$ & $1 / 9$ & $1 / 9$ & $1 / 9$ & $1 / 9$ & $1 / 9$
\end{tabular}

Тогда на множестве $X_{H}, X_{L}$ получаем вероятности $\{1 / 3,1 / 3,1 / 3\}$ и $\{5 / 18,7 / 18,1 / 3\}$ соответственно. Заметим, что распределения вероятностей на $X_{H}, X_{L}$ являются зависимыми, так как, например,

$$
\frac{1}{18}=\mathbf{P}(1,0) \neq \mathbf{P}_{H}(1) \mathbf{P}_{L}(0)=\frac{1}{3} \cdot \frac{5}{18}
$$

Функцию $\delta$ зададим так, чтобы выполнялось первое условие определения 3. Функцию $\lambda\left(\left(x_{H}, x_{L}\right),\left(s_{H}, s_{L}\right)\right)=\left(y_{H}, y_{L}\right)$ зададим таблицей

\begin{tabular}{c|ccccccccc}
$\left(x_{H}, x_{L}\right)$ & $(0,0)$ & $(0,1)$ & $(1,0)$ & $(1,1)$ & $(0, e)$ & $(e, 0)$ & $(1, e)$ & $(e, 1)$ & $(e, e)$ \\
\hline$\left(s_{H}^{1}, s_{L}^{1}\right)$ & $(0,0)$ & $(0,0)$ & $(1,0)$ & $(1,0)$ & $(0, e)$ & $(e, 0)$ & $(1, e)$ & $(e, 0)$ & $(e, e)$ \\
$\left(s_{H}^{1}, s_{L}^{2}\right)$ & $(0,1)$ & $(0,0)$ & $(1,1)$ & $(1,0)$ & $(0, e)$ & $(e, 1)$ & $(1, e)$ & $(e, 0)$ & $(e, e)$ \\
$\left(s_{H}^{2}, s_{L}^{1}\right)$ & $(1,0)$ & $(1,0)$ & $(0,0)$ & $(0,0)$ & $(1, e)$ & $(e, 0)$ & $(0, e)$ & $(e, 0)$ & $(e, e)$ \\
$\left(s_{H}^{2}, s_{L}^{2}\right)$ & $(1,1)$ & $(1,0)$ & $(0,1)$ & $(0,0)$ & $(1, e)$ & $(e, 1)$ & $(0, e)$ & $(e, 0)$ & $(e, e)$
\end{tabular}

Из нее видно, что если фиксированы вход $x_{L} \in X_{l}$ и состояние $s_{L} \in S_{L}$, то выход $y_{L}$ не меняется при любых $x_{H} \in X_{H}$ и $s_{H} \in S_{H}$, то есть выполнено второе условие определения 3. Таким образом, учитывая, как мы выбрали функцию $\delta$, видим, что пользователь $L$ не видит $H$. 
Теперь найдем распределения вероятностей

$$
\mathbf{Q}_{L}^{s_{L}}\left(y_{L}\right)=\mathbf{Q}_{L}^{s^{\prime}}\left(y_{L}\right), \quad s^{\prime}=\left(s_{H}, s_{L}\right), \quad s_{H} \in S_{H},
$$

и условные вероятности $\mathrm{P}^{s}\left(y_{L} \mid x_{H}\right)$. Пользуясь таблицей, формулами (5), (7), (6), а также леммами $1,2,3$ и следствием 1 , находим, что

$$
\begin{aligned}
& \mathbf{Q}_{L}^{s_{L}^{1}}(0)=\mathbf{P}(\{(0,0),(0,1),(1,0),(1,1),(e, 0),(e, 1)\})=2 / 3, \\
& \mathbf{Q}_{L}^{s_{L}^{1}}(1)=\mathbf{P}(\{\varnothing\})=0, \\
& \mathbf{Q}_{L}^{s_{L}^{1}}(e)=\mathbf{P}(\{(0, e),(1, e),(e, e)\})=1 / 3 \\
& \mathbf{Q}_{L}^{s_{L}^{2}}(0)=\mathbf{P}(\{(0,1),(1,1),(e, 1)\})=7 / 18, \\
& \mathbf{Q}_{L}^{s_{L}^{2}}(1)=\mathbf{P}(\{(0,0),(1,0),(e, 0)\})=5 / 18, \\
& \mathbf{Q}_{L}^{s_{L}^{2}}(e)=\mathbf{P}(\{(0, e),(1, e),(e, e)\})=1 / 3 .
\end{aligned}
$$

Совместную вероятность $\mathbf{P}^{s}\left(x_{H}, y_{L}\right)$ задает таблица

\begin{tabular}{c|ccccccccc}
$\left(x_{H}, y_{L}\right)$ & $(0,0)$ & $(0,1)$ & $(1,0)$ & $(1,1)$ & $(0, e)$ & $(e, 0)$ & $(1, e)$ & $(e, 1)$ & $(e, e)$ \\
\hline$\left(s_{H}^{1}, s_{L}^{1}\right)$ & $2 / 9$ & 0 & $2 / 9$ & 0 & $1 / 9$ & $2 / 9$ & $1 / 9$ & 0 & $1 / 9$ \\
$\left(s_{H}^{1}, s_{L}^{2}\right)$ & $1 / 9$ & $1 / 9$ & $1 / 6$ & $1 / 18$ & $1 / 9$ & $1 / 9$ & $1 / 9$ & $1 / 9$ & $1 / 9$ \\
$\left(s_{H}^{2}, s_{L}^{1}\right)$ & $2 / 9$ & 0 & $2 / 9$ & 0 & $1 / 9$ & $2 / 9$ & $1 / 9$ & 0 & $1 / 9$ \\
$\left(s_{H}^{2}, s_{L}^{2}\right)$ & $1 / 9$ & $1 / 9$ & $1 / 6$ & $1 / 18$ & $1 / 9$ & $1 / 9$ & $1 / 9$ & $1 / 9$ & $1 / 9$
\end{tabular}

Условные вероятности можно найти по формуле

$$
\mathbf{P}^{s_{L}}\left(y_{L} \mid x_{H}\right)=\frac{\mathbf{P}^{\left(s_{H}, s_{L}\right)}\left(x_{H}, y_{L}\right)}{\mathbf{P}_{H}\left(x_{H}\right)}=3 \mathbf{P}^{\left(s_{H}, s_{L}\right)}\left(x_{H}, y_{L}\right) .
$$

Из значений $\mathbf{Q}^{s_{L}}\left(y_{L}\right)$ и $\mathbf{P}^{s}\left(x_{H}, y_{L}\right)$, приведенных в предыдущей таблице, видно, что определение 4 невидимости по вероятности выполняется, так как, например,

$$
1 / 2=\mathbf{P}^{\left(s_{H}, s_{L}^{2}\right)}(0 / 1) \neq \mathbf{Q}_{L}^{s_{L}^{2}}(0)=7 / 18,
$$

для всех $s_{H} \in S_{H}$.

Данный пример показывает, что невидимость в смысле определения 3 не гарантирует невидимости по вероятности.

Легко привести достаточные условия невидимости по вероятности.

Теорема 1. Пусть L не видит H. Тогда для невидимости по вероятности достаточно, чтобы выполнялосъ равенство

$$
\mathbf{P}\left(x_{H}, x_{L}\right)=\mathbf{P}_{H}\left(x_{H}\right) \mathbf{P}\left(x_{L}\right)
$$

$\partial \Omega я$ всех $x \in X$.

Доказателъство. Из определения невидимости следует, что

$$
\begin{aligned}
\mathbf{P}^{s}\left(y_{L} / x_{H}\right) & =\frac{1}{\mathbf{P}_{H}\left(x_{H}\right)} \mathbf{P}\left\{\left(x_{H}, x_{L}\right) \in X: \lambda\left(\left(x_{H}, x_{L}\right),\left(s_{H}^{1}, s_{L}\right)\right)=\left(y_{H}, y_{L}\right), y_{H} \in Y_{H}\right\} \\
& =\sum_{\left(x_{H}, x_{L}\right)} \frac{\mathbf{P}\left(x_{H}, x_{L}\right)}{\mathbf{P}\left(x_{H}\right)}
\end{aligned}
$$


где суммирование ведется по всем парам $\left(x_{H}, x_{L}\right) \in X$ таким, что

$$
\left.\lambda\left(\left(x_{H}, x_{L}\right),\left(s_{H}^{1}, s_{L}\right)\right)=\left(y_{H}, y_{L}\right), y_{H} \in Y_{H}\right\}
$$

Отсюда в силу независимости получаем, что

$$
\mathbf{P}^{s}\left(y_{L} \mid x_{H}\right)=\sum_{y_{H} \in Y_{H}} \mathbf{P}\left\{x \in X: \lambda(x, s)=\left(y_{H}, y_{L}\right)\right\}=\mathbf{Q}_{L}^{s}\left(y_{L}\right) .
$$

Теорема доказана.

\section{Список литературы}

1. Moskowitz I. S., Costich O. L., A classical automata approach to noninterference type problems. Proc. Computer Security Foundations Workshop 5. IEEE Press, 1992, 2-8.

Статья поступила 31.08.2001. 